\title{
The TBP gene from Aspergillus nidulans - structure and expression in Saccharomyces cerevisiae
}

\author{
Robert Kucharski ${ }^{1}+$ and Ewa Bartnik ${ }^{1,2}$
}

Department of Genetics, University of Warsaw ${ }^{1}$, and Institute of Biochemistry and Biophysics, Polish Academy of Sciences ${ }^{2}$ Pawińskiego 5a, 02-106 Warsaw, Poland
Author for correspondence: Ewa Bartnik. Tel: +48 226597072 ext. 2247. Fax: +48 226584754. e-mail: ebartnik@ibbrain.ibb.waw.pl

The genomic and CDNA copy of the TATA-binding protein (TBP) gene from the filamentous fungus Aspergillus nidulans have been cloned. The gene is interrupted by four introns, one of which is in the long $5^{\prime}$ untranslated region of $615 \mathrm{bp}$. The transcription initiation site was established and the levels of MRNA were analysed under diverse growth conditions and found to vary severalfold. The gene encodes a protein of 268 amino acids composed of an $\mathbf{N}$ terminal domain of 88 amino acids with no significant homology to other TBPs and a C-terminal domain of 180 amino acids with about $95 \%$ homology to other fungal TBPs. A CDNA clone under the yeast ADH1 promoter was able to substitute for the yeast TBP gene in vivo; however, the transformants obtained grew poorly at $35^{\circ} \mathrm{C}$ and on galactose and glycerol at $30^{\circ} \mathrm{C}$, though they could grow in the presence of copper ions or aminotriazole at this temperature. This phenotype may be the result of altered function of $A$. nidulans TBP in certain yeast transcription activation pathways.

Keywords: Aspergillus nidulans, TBP

\section{INTRODUCTION}

Since the cloning of the first gene encoding TATAbinding protein (TBP) from Saccharomyces cerevisiae, the TBP genes (or more often cDNAs) have been cloned from a number of organisms. General comparisons indicate a strong conservation of the C-terminal region of the TBP protein with a considerable variation of the N-terminal region. The crystallographic (threedimensional) structure of the protein-DNA complex has been analysed and a lot is known about the interactions of TBP with various general and specific transcription factors such as TAFs, TFIIB, E1A and others (for a review see Hernandez, 1993).

For many years, the filamentous fungus Aspergillus nidulans has served as a model organism for studying gene structure and expression. The detailed regulation of many genes has been analysed, and the genes for a number of specific transcriptional regulators have been

\footnotetext{
†Present address: Visual Sciences, RSBS, The Australian National University, Canberra ACT 0200, Australia.

Abbreviations: TBP, TATA-binding protein; uORF, upstream open reading frame; UTR, untranslated transcribed region.

The GenBank accession numbers for the nucleotide sequences reported in this paper are U28332 (CDNA) and U28333 (gene).
}

cloned and sequenced. So far, however, there are no data on structure and interactions of the components of its basic transcriptional apparatus. Moreover, no TBP gene sequence from a filamentous fungus has been published. We therefore started the analysis of $A$. nidulans transcription initiation complexes from the isolation and characterization of the TBP gene.

\section{METHODS}

Plasmids and DNA libraries. pBluescript $\mathrm{KS}(+)$ was from Stratagene and the $S$. cerevisiae shuttle vector pL361 was obtained from Dr T. Vernet (McGill University, Canada). The pIC19R plasmid containing a fragment of the A. nidulans actin gene was obtained from Dr R. Bradshaw (Massey University, Palmerston North, New Zealand).

The $A$. nidulans genomic DNA library in $\lambda$ EMBL3 was obtained from Dr C. Roberts (Leicester University, UK). It contains $7-20 \mathrm{~kb}$ DNA fragments obtained by partial Sau3A digestion cloned in the $B a m H I$ site of the vector. The A. nidulans cDNA library in $\lambda$ ZAP was purchased from the Fungal Genetics Stock Center (University of Kansas Medical Center, USA).

Organisms and growth conditions. The following strains were used: Escherichia coli DH5 $\alpha$ (Sambrook et al., 1989) and XL-1 Blue (Stratagene), S. cerevisiae SHY67 (Hahn et al., 1989), A. nidulans proA6 pabaA9 biA1 suC6 and the proto- 
trophic $y$ strain (Department of Genetics, University of Warsaw).

The organisms were grown on solid and liquid media: for $E$. coli, LB and $2 \times$ TY; for S. cerevisiae, YPD or YNB (with supplements as required; Sambrook et al., 1989). Medium containing 5-fluoroorotic acid for plasmid shuffling was according to Boeke et al. (1987). Carbon sources were used at $2 \%$. Copper sulphate was added up to a $0.25 \mathrm{mM}$ concentration and $50 \mathrm{mM}$ 3-amino-1,2,4-triazole was used. $A$. nidulans was grown at $37^{\circ} \mathrm{C}$ on $\mathrm{CM}$ or $\mathrm{MM}$ medium (Pontecorvo et al., 1953) supplemented with a carbon source (glycerol or glucose) at $1 \%$ and nitrogen sources (glutamine, sodium nitrate or arginine) at $10 \mathrm{mM}$. Heat shock was performed at $55^{\circ} \mathrm{C}$ for $15 \mathrm{~min}$ in standard MM.

Molecular biology techniques. E. coli was transformed by the standard calcium chloride method (Sambrook et al., 1989). Yeast cells were transformed according to Chen et al. (1992).

Nucleic acid isolation and manipulation were performed according to Sambrook et al. (1989) unless stated otherwise.

A. nidulans DNA was prepared according to Yelton et al. (1985).

RNA from A. nidulans was prepared using the TRIzol reagent (BRL). poly(A) RNA was purified by single-step chromatography on oligo-dT cellulose (Sigma).

Restriction endonucleases and ligase were from Amersham and MBI Fermentas and were used according to the manufacturers' instructions.

DNA fragments were purified after excision from gels using electroelution in the Bio-Rad model 422 apparatus.

DNA electrophoresis was performed in standard Tris-acetate buffer using $0 \cdot 3-3 \%$ agarose (Sigma type II). RNA was analysed on denaturing formaldehyde gels. The molecular mass markers were $\lambda$ DNA cleaved with BstEII (MBI Fermentas), the $1 \mathrm{~kb}$ ladder (BRL) and the RNA Marker $0 \cdot 36-9.5 \mathrm{~kb}$ (Sigma).

DNA fragments were labelled using $\left[\alpha^{32} \mathrm{P}\right] \mathrm{dATP}$ (Amersham) and the Megaprime kit (Amersham). Nested deletions for sequencing by the dideoxy method were made using the Pharmacia double-stranded Nested Deletions kit. Sequencing was done using Sequenase version 2.0 (USB) or Taq polymerase (MBI Fermentas or Promega). Sequencing and primer extension products were analysed on $6 \%$ polyacrylamide gels containing $50 \%$ urea.

Primer extension was performed according to de Graaff et al. (1994) with some modifications, using Superscript II RTase (BRL) and $10 \mu \mathrm{g}$ total RNA.

PCR. PCR reactions were performed in a Perkin Elmer Thermocycler. The primers were degenerate 20-mers corresponding to NAEYNPK ( 5 ' primer 'TBPFOR') and FVSGIV ( 3 ' primer 'TBPREV'). They were added at $20 \mathrm{pmol}$ each to $1 \mu \mathrm{g}$ genomic DNA. Initial denaturation was for $5 \mathrm{~min}$ at $94^{\circ} \mathrm{C}$. Cycling conditions were five cycles of low stringency (denaturation for $1 \mathrm{~min}$ at $92^{\circ} \mathrm{C}$; annealing at $35^{\circ} \mathrm{C}$; ramping for $2.5 \mathrm{~min}$ at $72{ }^{\circ} \mathrm{C}$; elongation for $3 \mathrm{~min}$ at $72{ }^{\circ} \mathrm{C}$ ) followed by 45 moderate stringency cycles (denaturation for $1 \mathrm{~min}$ at $92^{\circ} \mathrm{C}$; annealing for $1 \mathrm{~min}$ at $42^{\circ} \mathrm{C}$; elongation for $3 \mathrm{~min}$ at $72^{\circ} \mathrm{C}$ ). Each cycle was $5 \mathrm{~s}$ longer than the previous cycle. The reaction products were purified on a Centricon 30 concentrator (Amicon). Products were cloned in the T-tailed vector prepared from pBluescript $\mathrm{KS}(+)$ according to Marchuk et al. (1991).
Hybridization. Hybond N (Amersham) was used for all blotting procedures and hybridizations were performed in a Hybaid mini-oven using standard solutions. The Southern genomic hybridizations were performed for $24 \mathrm{~h}$ at $65^{\circ} \mathrm{C}$ in $3 \times$ SSC with $0.25 \%$ lyophilized skim milk. After the hybridization, the filters were washed four times for $10 \mathrm{~min}$ in $3 \times$ SSC, $0 \cdot 1 \%$ SDS at room temperature and then for $5 \mathrm{~min}$ at $65^{\circ} \mathrm{C}$ in $0.1 \times \mathrm{SSC}, 0.1 \%$ SDS. After hybridization either standard autoradiography was performed or the filters were used to expose phosphor storage screens and the images were analysed using a PhosphorImager SF (Molecular Dynamics).

Sequence analysis. Sequence homologies were analysed using BLAST (Altschul et al., 1990). Multiple sequence alignments were performed with the CLusTal $\mathrm{v}$ package (Higgins \& Sharp, 1988). PHYLIP (Felsenstein, 1989) was used to derive a phylogenetic tree. The algorithm described by Zuker (1989) was used to analyse potential RNA conformation. The ImageQuant version 3.3 program (Molecular Dynamics) was used for quantification of hybridization signals.

\section{RESULTS AND DISCUSSION}

The TBP C-terminal domain of 180 amino acids is strongly conserved and the degenerate oligonucleotide primers for PCR were designed on the basis of alignment of sequences from seven organisms. We did not attempt to use a heterologous probe as preliminary data (P. P. Stępień, personal communication) indicated that no hybridization of the S. cerevisiae TBP gene to A. nidulans DNA would be obtained. The PCR primers (degenerate 20 -mers) corresponded to NAEYNPK $\left(5^{\prime}\right.$ primer 'TBPFOR') and FVSGKIV ( 3 ' primer 'TBPREV') sequences of the C-terminal domain.
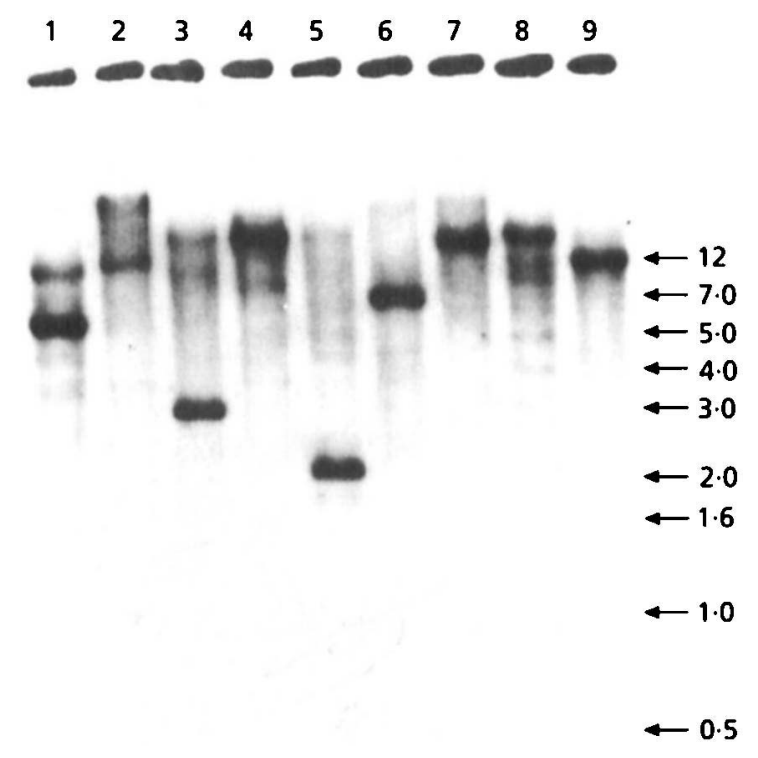

Fig. 1. Genomic hybridization of DNA restriction fragments of $A$. nidulans with the AnTBPG probe - a fragment of the TBP coding sequence of $A$. nidulans obtained using PCR. Lanes 1-9 show a Southern blot of genomic DNA digested with: 1, EcoRV; 2, BamHI; 3, Bg/ll; 4, EcoRI; 5, Hindlll; 6, Pstl; 7, Sall; 8, Xbal; 9, $X$ hol. The molecular mass marker is the $1 \mathrm{~kb}$ ladder (BRL). 


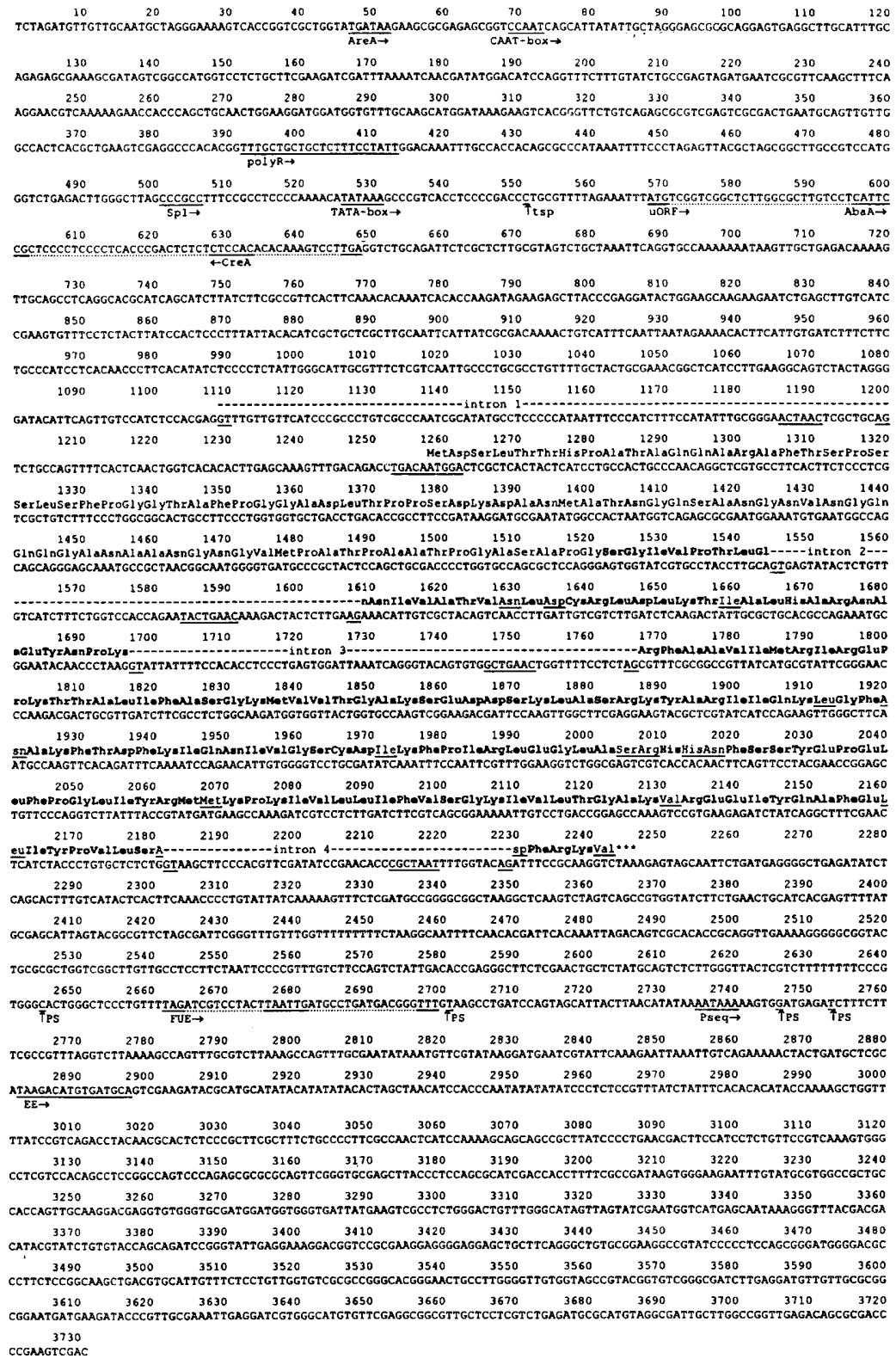

\begin{abstract}
Fig. 2. Sequence of the $A$. nidulans TBP gene. Transcriptional, splicing and translational regulatory sequences are marked. The C-terminal domain amino acids are in bold and those which are different in comparison to $S$. cerevisiae are underlined. tsp, transcriptional starting point; polyR, polypyrimidine tract; $P S$, polyadenylation site; FUE, 'far upstream' element (transcription termination signal); Pseq, mammalian consensus polyadenylation sequence; $\mathrm{EE}$, efficiency element (transcription termination signal). Arrows denote the orientation of regulatory sequences.
\end{abstract}

The designed primers were used for PCR of $A$. nidulans genomic DNA and a fragment larger than expected was obtained (approx. $440 \mathrm{bp}$ instead of $368 \mathrm{bp}$ ). The genome-amplified PCR fragment (designated AnTBPG) was cloned and sequenced. A high homology to known sequences encoding TBP was shown for 327 bases and the highest homology was found to $S$. cerevisiae and Schizosaccharomyces pombe sequences. The $70 \mathrm{bp}$ fragment which showed no homology to any known sequences was assumed to be an intron or its fragment, as sequences characteristic of $A$. nidulans introns were detected in it (data not shown). In order to confirm that the amplified sequence is derived from $A$. nidulans, the AnTBPG probe was hybridized under stringent conditions to a Southern blot of A. nidulans DNA digested with a number of restriction endonucleases. Single strongly hybridizing restriction fragments were detected for a number of restriction enzymes (Fig. 1). This result proves that $A$. nidulans TBP coding fragments had been amplified, and that there is only one copy of the TBP coding gene in the genome. This is typical of most eukaryotes, although two copies have been found in Arabidopsis and in other plants (Vogel et al., 1993).

The AnTBPG probe was used to screen an A. nidulans genomic library in the $\lambda E M B L 3$ vector. One clone, $\lambda$ TBPG5.11, was subjected to detailed restriction and hybridization analysis. A $3.7 \mathrm{~kb}$ XbaI-SalI fragment containing the TBP sequence was subcloned into pBluescript $\mathrm{KS}(+)$. The plasmid obtained was designated pAnTBPG1. The cDNA library was screened using the AnTBPG probe and after preliminary restriction mapping of plasmids rescued from 18 clones the largest plasmid was selected for further analysis; it 


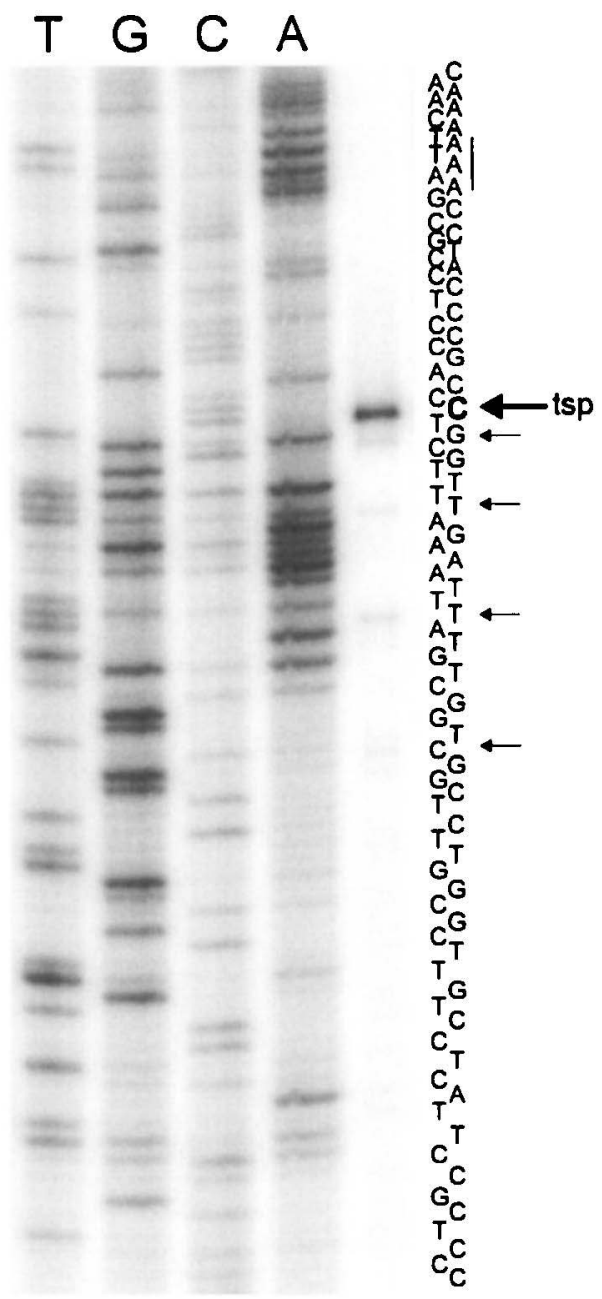

Fig. 3. Primer extension analysis of the transcription initiation site. Digital image from Phosphorlmager. The analysis of products of reverse transcription of total $A$. nidulans RNA using a primer complementary to nucleotides $644-663$ is shown. The products of PCR sequencing of the PAnTBPG1 plasmid using the same primer are shown in the first four lanes. The letters denote the nucleotides used for termination in sequencing reactions. tsp, transcriptional initiation site; arrows denote potential minor start sites.

contained a cDNA fragment corresponding in size to the A. nidulans TBP mRNA which was found to be $1.9 \mathrm{~kb}$ in size (data not shown).

The genomic fragment and eight different $\mathrm{cDNA}$ clones were sequenced on both strands using plasmid templates with nested deletions prepared with ExoIII and S1 nucleases and the results are presented in Fig. 2.

The transcription initiation site was determined by the primer extension method using a primer complementary to nucleotides $644-663$. On the denaturing gel one predominant product was found (Fig. 3) and the main transcription initiation site was at nucleotide 551 .

The structure of the TBP gene is typical of most genes of filamentous fungi. It is composed of five exons and four introns. All introns have consensus sequences and exon-intron junctions similar to the consensus occurring in introns of filamentous fungi $\left(5^{\prime}\right.$ WG/GTRNGYY...YGCTAAC...YAG/RY 3') (Ballance, 1986; Gurr et al., 1987). Three of the introns have a size which is typical of most $A$. nidulans introns (up to 80 nucleotides). The first intron is slightly longer ( 92 nucleotides) and is moreover located in the $5^{\prime}$ UTR of the transcript. This is the second known example of an intron in an $A$. nidulans sequence which is not translated, as a 140 nucleotide intron has been found in the $5^{\prime}$ UTR of the facA gene encoding acetyl-CoA synthetase (Connerton et al., 1990). The strong conservation of introns in areas which do not undergo translation may play a regulatory role in the expression of genes in plants and vertebrates (Pearson \& Meagher, 1990) but the mechanism has only been described in detail for the leader of the gene encoding human $\beta$-actin containing an enhancer which binds a factor required for efficient transcription (Kawamoto et al., 1988). The second and the third introns are found in strongly conserved positions, as in other TBP genes, which may reflect the structure of the ancestral gene (compare Nakashima et al., 1995).

The mature TBP mRNA in A. nidulans has a very long $5^{\prime}$ UTR of 615 nucleotides which contains an ORF encoding a peptide of 26 amino acids at the very $5^{\prime}$ end (Fig. 2). In general, the length of the leader sequences in A. nidulans mRNAs does not exceed 200 nucleotides and sequences longer than 500 nucleotides are exceptional (Gurr et al., 1987). Three other examples of this type of long leader sequence are known: in the stu $\mathrm{A}$ gene (1088 nucleotides; Miller et al., 1991), the creA gene (590 nucleotides; Dowzer \& Kelly, 1991) and the bim $G$ gene (885 nucleotides; Doonan \& Morris, 1989). All these genes encode regulatory proteins. Moreover, the 5' UTR of the TBP gene shows a significant degree of sequence homology to the leader of the $\operatorname{bim} G$ gene, which also contains an uncharacterized short ORF encoding a potential peptide of 34 amino acids (data not shown). In addition, computer analysis revealed that the 5 ' UTR has regions with a strong tendency to form secondary structure, an especially stable structure $\left[\Delta G_{0}=-27.7 \mathrm{kcal} \mathrm{mol}^{-1}\left(-116 \cdot 3 \mathrm{~kJ} \mathrm{~mol}^{-1}\right)\right]$ can form on the segment $245-450$ nucleotides downstream from the transcription initiation site.

The function of the ORF in the mRNA leader (uORF, upstream open reading frame) is unknown. According to Kozak (1987), the presence of two cistrons in eukaryotic mRNA does not prevent translational reinitiation in the second one if the first cistron has a limited length and both are sufficiently far apart. The uORF may decrease the rate of initiation of translation and cause increased transcript degradation (Oliviera \& McCarthy, 1995). Two models explaining the role of these UORFs exist: the first proposes a decrease of the efficiency of translation of the proper protein by a decrease in the rate of ribosome movement due to uORF translation (ribosome stall model; Damiani \& Wessler, 1993) and the second postulates a repressor function of the uORF-encoded peptide, which, by a specific in- 
teraction with the ribosome, a termination factor or RNA, causes a blocking of the mRNA $5^{\prime}$ end (peptide repressor model; Hill \& Morris, 1993). The secondary structure can additionally act as a negative translation regulation signal since in fungi such structures occurring near the initiation codon may stop the small ribosome subunit from scanning (Vega Laso et al., 1993).

Analysis of codon usage was performed using the relative synonymous codon usage (RSCU) index (Sharp et al., 1986). In the TBP gene, optimal codons are the most common, but for valine, asparagine, glutamic acid, aspartic acid and serine, codons which very rarely occur in A. nidulans genes are used. On the basis of our analysis, the TBP mRNA may be considered as a transcript with a moderate translational efficiency. Most genes encoding $A$. nidulans regulatory proteins show a lack of preference in codon usage (Lloyd \& Sharp, 1991).

The promoter of the analysed gene contains many elements characteristic of genes of higher eukaryotes. These include the CAAT box, a polypyrimidine tract, the $\mathrm{Sp} 1$ recognition sequence and a consensus TATA box (Fig. 2). This distinguishes the A. nidulans TBP gene from most fungal genes, which rarely contain all these elements simultaneously (Gurr et al., 1987). The TATA box occurs at the -25 position characteristic of the promoters of most eukaryotic genes transcribed by RNA polymerase II. Even though relatively few transcription factors have been analysed from A. nidulans, in fungi in general many homologues of mammalian transcription factors are known (Dhawale \& Lane, 1993). The presence of the above mentioned regulatory sequences in the TBP gene promoter may therefore be considered significant for transcriptional control. Three sequences found in the vicinity of the transcription initiation site are known to be recognized by specific factors involved in general regulation of $A$. nidulans metabolism (CreA - carbon repression; AreA - nitrogen repression; and $\mathrm{AbaA}$ - involved in regulation of conidiophore development).

The presence of both potential binding sites for positive transcriptional regulators (AreA, etc.) in the vicinity of the TBP transcriptional initiation site and of negative regulatory elements in the $5^{\prime}$ end of the mRNA suggests that a complicated interplay may occur between processes at the level of initiation of transcription and translation to maintain an optimal TBP level and prevent the formation of non-functional complexes between excess TBP protein and other components of the transcriptional machinery.

Regulation of TBP transcription by main regulatory circuits such as carbon and nitrogen repression was analysed, as were the effects of heat shock and stage of development. The amount of the specific transcript per cell was found to be very low, and poly(A) RNA had to be used for all the experiments. A probe for detecting $A$. nidulans actin mRNA was used to standardize the amounts of RNA present in each sample. TBP mRNA levels were found to vary depending on the culture conditions, especially depending on the nitrogen source used. The transcript level also increased after incubation at $55^{\circ} \mathrm{C}$. We were unable to isolate sufficient amounts of poly(A) RNA from mycelium subjected to starvation to analyse the level of transcripts for TBP and actin. Analysis of the levels of the TBP transcript in developing mycelium indicated a rapid increase in transcript amounts between 6 and $8 \mathrm{~h}$ after swelling the conidia in minimal medium. Insufficient material for detailed analysis was obtained from conidia and during germination ( $4 \mathrm{~h}$ after swelling). The regulation of mRNA levels by carbon and nitrogen source correlates well with the occurrence of potential binding sites for CreA and AreA near the transcription initiation site (data not shown).

In the $3^{\prime}$ region of the TBP gene sequences resembling the yeast 'far upstream' transcription termination element (Zaret \& Sherman, 1982; nucleotides 2662-2699), the mammalian polyadenylation signal (nucleotides $2735-2740$ ) and a sequence $70 \%$ homologous to the efficiency element (EE) of Schiz. pombe (Humphrey et al., 1994; nucleotides 2882-2897) have been found. On the basis of the genomic DNA sequence as well as that of the eight cloned cDNAs analysed, the existence of four alternative polyadenylation sites in the vicinity of various signals can be postulated. The polyadenylation site after position 2701 of the gene is used most often and is located beyond the sequence homologous to one of the $S$. cerevisiae transcription termination signals. The existence of multiple polyadenylation sites is characteristic of many genes of filamentous fungi (Gurr et al., 1987).

The initiation codon for TBP was determined on the basis of the consensus sequences determined for various organisms (Kozak, 1991) and A. nidulans (R. Kucharski, unpublished data). Only the AUG codon at position 1258 is located within a consensus sequence with an adenine at -3 (the $A$. nidulans consensus sequence is $5^{\prime}$ YCANNATGNNY $3^{\prime}$ ). The presence of the consensus sequence and the distinct homology of the amino acid sequence to the N-terminus of the Schiz. pombe TBP allowed the assignment of the first TBP codon in $A$. nidulans with a high degree of certainty.

The A. nidulans C-terminal TBP domain of 180 amino acids has $95 \%$ identity to corresponding domains in other fungi. In the $\mathrm{N}$-terminal domain of 88 amino acids, only one similar block can be found in another $\mathrm{N}$ terminal domain - that of Schiz. pombe (PATAQ block; Fig. 4). In addition, there are two short arrays of simple sequences (CAR) encoding blocks of two and three glutamines. Comparison of the A. nidulans and $S$. cerevisiae TBP C-terminal domain sequences indicates that most differences are located in polymorphic positions. A number of amino acid substitutions can be found in the second repeat, especially helices $\mathrm{H}^{\prime}$ and $\mathrm{H} 2^{\prime}$. As these amino acids are exposed on the protein surface (Chasman et al., 1993) they may participate in protein-protein interactions. The putative regulatory role of TBP N-terminal domains is unclear. They 
(a)

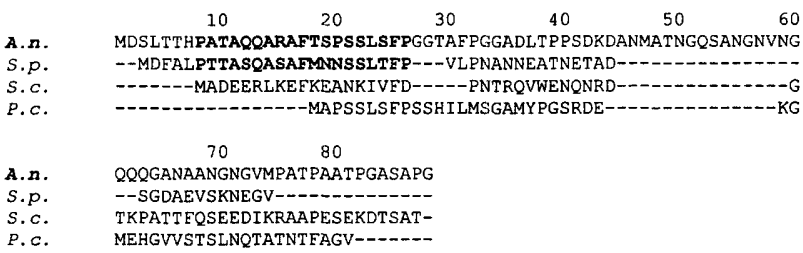

(b)

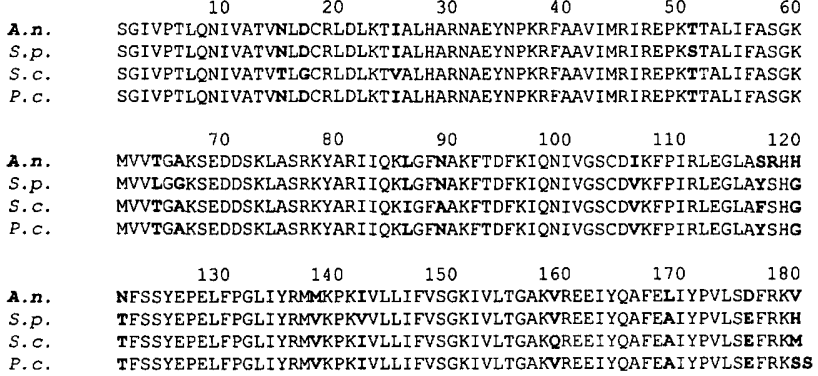

Fig. 4. Alignment of $\mathrm{N}$-terminal (a) and C-terminal (b) domains of fungal TBPs: A. nidulans (A.n.), Schiz. pombe (S.p.; Fikes et al., 1990), S. cerevisiae (S.C.; Hahn et al., 1989) and Pneumocystis carinii, prototype (P.c.; Sunkin \& Stringer, 1995) The PATAQ block and similar amino acids in the C-terminal domains are emphasized in bold.

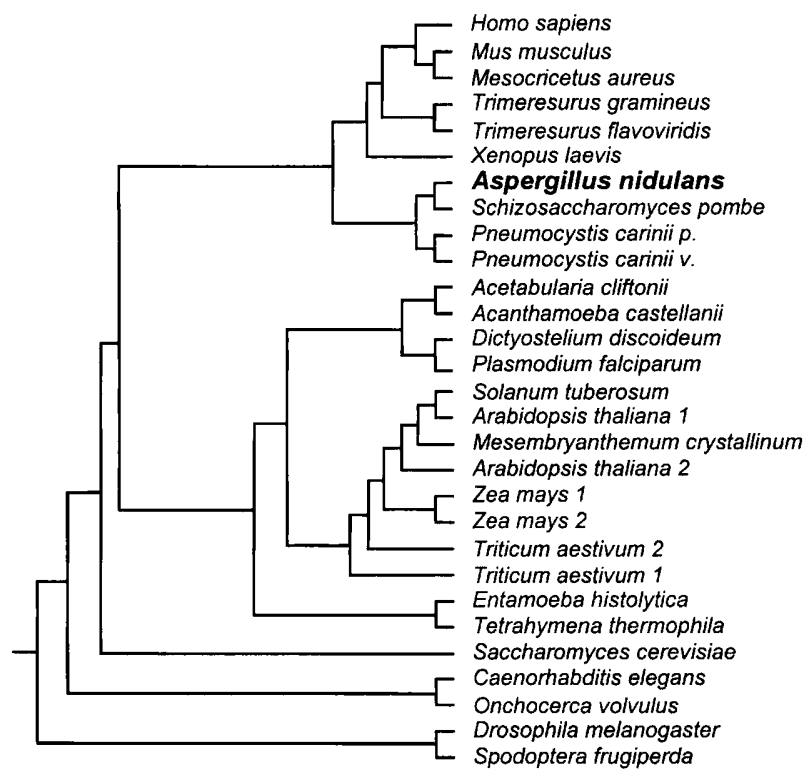

Fig. 5. Phylogenetic tree of amino acid sequences of C-terminal TBP domains obtained by using the following programs: CLUSTALW (sequence comparison), PROTDIST (creation of distance matrices), NEIGHBOR (obtaining tree structure by neighbour joining) and DRAWGRAM (drawing of a rooted tree). The lengths of the branches do not correspond to the frequencies of amino acid substitutions.

undergo an independent evolution and differ both in length and sequence between different organisms (Hernandez, 1993). The existence of stretches of

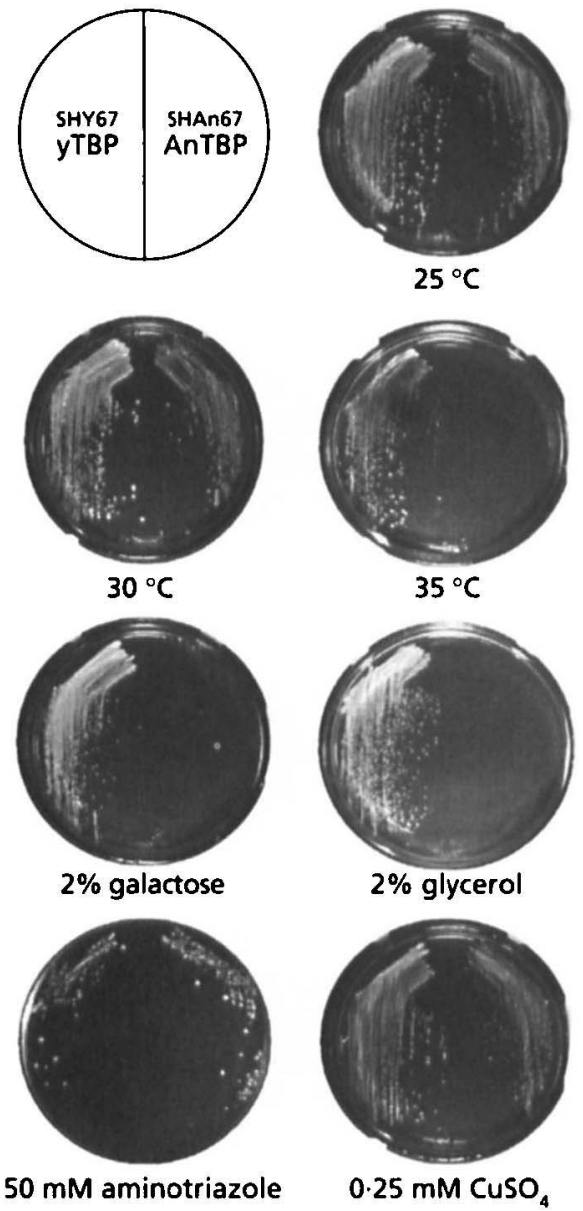

Fig. 6. Growth tests of $S$. cerevisiae expressing the $A$. nidulans TBP compared to the control strain grown at $30^{\circ} \mathrm{C}$ on YPD media containing galactose or glycerol and on YNB mineral media containing aminotriazole and copper ions and YPD/glucose medium at $35^{\circ} \mathrm{C}$. Digital camera picture.

glutamines encoded by simple repetitive sequences, e.g. (YRR $)_{n}$, is characteristic of the TBP N-terminal domains of higher eukaryotes (Hancock, 1993) and may be the effect of a base slippage phenomenon. A phylogenetic tree based on the N-terminal domain sequence is shown in Fig. 5.

As there is no easy system of analysing in vivo or in vitro expression of $A$. nidulans genes (no in vitro transcription system has been established and there are essentially no non-integrating vectors for transformation), we introduced the TBP cDNA under the control of the $S$. cerevisiae $A D H 1$ promoter in the shuttle vector pL361 into $S$. cerevisiae strain SHY87, which allows for the selection of transformants bearing only the recombinant A. nidulans TBP sequences and deprived of the yeast TBP gene using the plasmid-shuffling method (Hahn et al., 1989). The $A D H 1$ promoter is a strong constitutive promoter frequently used for this type of experiment (Fikes et al., 1990). As viable yeast cells were obtained, this indicates that the $A$. nidulans TBP can substitute all essential $S$. cerevisiae TBP functions. However, in 
contrast to cells containing the $S$. cerevisiae TBP, cells with $A$. nidulans TBP grew poorly at $35^{\circ} \mathrm{C}$ (Fig. 6). So far, Schiz. pombe (Fikes et al., 1990) and two variants of maize TBP (Vogel et al., 1993) have been found to substitute for the $S$. cerevisiae TBP, as did human TBP after changing the 231st amino acid from arginine to lysine (Cormack et al., 1994).

$S$. cerevisiae cells containing the A. nidulans TBP grew very poorly on glycerol and galactose as a source of carbon but were insensitive to $0.25 \mathrm{mM}$ copper sulphate and $50 \mathrm{mM}$ aminotriazole (Fig. 6). These results indicate that the $A$. nidulans protein probably cannot participate effectively in at least one process - that of Gal4 activation. On the other hand, resistance to copper ions and aminotriazole is normal, indicating that genes stimulated by Ace1 and Gcn 4 act normally. The lack of activation by Gal4 and normal activation by Ace1 and Gcn 4 may indicate that sequence differences between $A$. nidulans and $S$. cerevisiae TBP may cause incorrect behaviour of TBP during stimulation by Gal4. The result concerning the growth on galactose and copper resistance is of particular interest, as mutations analysed so far in yeast TBP (Stargell \& Struhl, 1995) caused a simultaneous lack of activation by three acidic activators - Gal4, Ace1 and Gcn4. This may indicate that there are distinct domains in TBP which are responsible for the interaction with different coactivators or for direct interaction with activating domains of Ace1 and Gal4. Even though a function in interaction with transcription factors has been ascribed to a number of the amino acids which are different between the two organisms, the effects of other differences occurring in C-terminal domains and also the influence of the $\mathrm{N}$-terminus of the A. nidulans TBP on the formation of fully functional preinitiation complexes in $S$. cerevisiae cells cannot be excluded. Our results may indicate the rapid coevolution of components of the transcriptional apparatus in eukaryotic micro-organisms.

\section{ACKNOWLEDGEMENTS}

We would like to thank Dr Steven Hahn for kindly providing yeast strain SHY67. This work was supported by grant nos BW-1154 and BW-1203/27 from the Faculty of Biology, University of Warsaw, and grant 6P04A04509 from the State Committee for Scientific Research. Help from the Foundation for Polish Science under the BIMOL Programme is also acknowledged.

\section{REFERENCES}

Altschul, S. F., Gish, W., Miller, W. \& Myers, E. W. (1990). Basic local alignment search tool. J Mol Biol 215, 403-410.

Ballance, D. J. (1986). Sequences important for gene expression in filamentous fungi. Yeast 2, 229-236.

Boeke, J. D., Trueheart, J., Natsoulis, G. \& Fink, G. R. (1987). 5Fluoorotic acid as a selective agent in yeast molecular genetics. Methods Enzymol 154, 164-175.

Chasman, D. I., Flaherty, K. M., Sharp, P. A. \& Kornberg, R. D. (1993). Crystal structure of yeast TATA-binding protein and model for interaction with DNA. Proc Natl Acad Sci USA 90, 8174-8178.

Chen, D. C., Yang, B. C. \& Kuo, F. T. (1992). One-step transformation of yeast in stationary phase. Curr Genet 21, 83-84.

Connerton, I. F., Fincham, J. R., Sandeman, R. A. \& Hynes, M. J. (1990). Comparison and cross-species expression of the acetyl coA synthetase genes of the ascomycete fungi, Aspergillus nidulans and Neurospora crassa. Mol Microbiol 4, 451-460.

Cormack, B. P., Strubin, M., Stargell, L. A. \& Struhl, K. (1994). Conserved and nonconserved functions of the yeast and human TATA binding proteins. Genes Dev 8, 1335-1343.

Damiani, R. D. \& Wessler, S. R. (1993). An upstream open reading frame represses expression of Lc, a member of the R/B family of maize transcriptional activators. Proc Natl Acad Sci USA 90, 8244-8248.

Dhawale, S. S. \& Lane, A. C. (1993). Compilation of sequence specific DNA binding proteins implicated in transcriptional control in fungi. Nucleic Acids Res 21, 5537-5546.

Doonan, J. H. \& Morris, N. R. (1989). The bimG gene of Aspergillus nidulans, required for completion of anaphase, encodes a homolog of mammalian phosphoprotein phosphatase 1. Cell 57, 987-996.

Dowzer, C. E. \& Kelly, J. M. (1991). Analysis of the creA gene, a regulator of carbon catabolite repression in Aspergillus nidulans. Mol Cell Biol 11, 5701-5709.

Felsenstein, J. (1989). PHYLIP - phylogeny inference package version 3. Cladistics 5, 164-166.

Fikes, J. D., Becker, D., Winston, F. \& Guarente, L. (1990). Striking conservation of TFIID in Schizosaccharomyces pombe and Saccharomyces cerevisiae. Nature 346, 291-294.

de Graaff, L. H., van de Broeck, H. C., Ooijen, A. J. J. \& Visser, J. (1994). Regulation of the xylanase encoding $x \ln A$ gene of Aspergillus tubigensis. Mol Microbiol 12, 479-490.

Gurr, S. J., Unkles, S. E. \& Kinghorn, J. R. (1987). The structure and organization of nuclear genes of filamentous fungi. In Gene Structure in Eukaryotic Microbes. Edited by J. R. Kinghorn. Oxford: IRL Press.

Hahn, S., Buratowski, S., Sharp, P. A. \& Guarente, L. (1989). Isolation of the gene encoding the yeast TATA binding protein TFIID : a gene identical to the SPT15 suppressor of Ty element insertions. Cell 58, 1173-1181.

Hancock, J. M. (1993). Evolution of sequence repetition and gene sequences in TATA-binding protein TBP (TFIID). Nucleic Acids Res 21, 2823-2830.

Hernandez, N. (1993). TBP, a universal eukaryotic transcription factor? Genes Dev 7, 1291-1308.

Higgins, D. G. \& Sharp, P. M. (1988). ClustaL: a package for performing multiple sequence alignment on a microcomputer. Gene 73, 237-244.

Hill, J. R. \& Morris, D. R. (1993). Cell-specific translational regulation of $\mathrm{S}$-adenosylmethionine decarboxylase mRNA. Dependence on translation and the coding capacity of the cis-acting upstream open reading frame. $J$ Biol Chem 268, 726-731.

Humphrey, T., Birse, C. E. \& Proudfoot, N. J. (1994). RNA $3^{\prime}$ end signals of the $S$. pombe ura 4 gene comprise a new site determining an efficiency element. EMBO J 13, 2441-2451.

Kawamoto, T., Makino, K., Niwa, H., Sugiyama, H., Kimura, S., Amemura, M., Nakata, A. \& Kakunaga, T. (1988). Identification of the human $\beta$-actin enhancer and its binding factor. Mol Cell Biol 8, 267-272. 
Kozak, M. (1987). Effects of intercistronic length on the efficiency of reinitiation by eukaryotic ribosomes. Mol Cell Biol 7, $3438-3445$.

Kozak, M. (1991). Structural features in eukaryotic mRNAs that modulate the initiation of translation. $J$ Biol Chem 266, 19867-19870.

Lloyd, A. T. \& Sharp, P. M. (1991). Codon usage in Aspergillus nidulans. Mol Gen Genet 230, 288-294.

Marchuk, D., Drumm, M., Saulino, A. \& Collins, F. S. (1991). Construction of T-vectors, a rapid and general system for direct cloning of unmodified PCR products. Nucleic Acids Res 19, 1154.

Miller, K. Y., Wu, J. \& Miller, B. L. (1991). stuA is required for cell pattern formation in Aspergillus. Genes Dev 6, 1770-1782.

Nakashima, K., Nobuhisa, I., Deshimaru, M., Ogawa, T., Shimoshigashi, Y., Fukumaki, Y., Hattori, M., Sakaki, Y., Hattori, S. \& Ohno, M. (1995). Structures of genes encoding TATA-box binding proteins from Trimesurus gramineus and T. flavoviridis snakes. Gene 152, 209-213.

Oliviera, C. C. \& McCarthy, J. E. (1995). The relationship between eukaryotic translation and mRNA stability. A short upstream open reading frame strongly inhibits translational initiation and greatly accelerates mRNA degradation in the yeast Saccharomyces cerevisiae. J Biol Chem 270, 8936-8943.

Pearson, L. \& Meagher, R. B. (1990). Diverse soybean actin transcripts contain a large intron in the $5^{\prime}$ untranslated leader: structural similarity to vertebrate muscle actin genes. Plant $\mathrm{Mol}$ Biol 14, 513-526.

Pontecorvo, G., Roper, J. A., Hemmons, L. M., MacDonald, R. D. \& Bufton, A. W. J. (1953). The genetics of Aspergillus nidulans. Adv Genetics 5, 141-238.
Sambrook, J., Fritsch, E. F. \& Maniatis, T. (1989). Molecular Cloning: a Laboratory Manual, 2nd edn. Cold Spring Harbor, NY: Cold Spring Harbor Laboratory.

Sharp, P. M., Tuohy, T. M. F. \& Mosurski, K. R. (1986). Codon usage in yeast: cluster analysis clearly differentiates highly and lowly expressed genes. Nucleic Acids Res 14, 5125-5143.

Stargell, L. A. \& Struhl, K. (1995). The TBP-TFIIA interaction in the response to acidic activators in vivo. Science $269,75-78$.

Sunkin, S. M. \& Stringer, J. R. (1995). Transcription factor genes from rat Pneumocystis carinii. J Eukaryot Microbiol 42, 12-19.

Vega Laso, M. R., Zhu, D., Sagliocco, F., Brown, A. J. P., Tuite, M. F. \& McCarthy, J. E. G. (1993). Inhibition of translational initiation in the yeast Saccharomyces cerevisiae as a function of the stability of hairpin structures in the mRNA leader. J Biol Chem 268, 6453-6462.

Vogel, J. M., Roth, B., Cigan, M. \& Freeling, M. (1993). Expression of two maize TATA binding protein genes and function of the encoded TBP proteins by complementation in yeast. Plant Cell $\mathbf{5}$, $1627-1638$.

Yelton, M. M., Timberlake, W. E. \& Van Den Hondel, C. A. M. J. J. (1985). A cosmid for selecting genes by complementation in Aspergillus nidulans: selection of the developmentally regulated yA locus. Proc Natl Acad Sci USA 82, 834-838.

Zaret, K. S. \& Sherman, F. (1982). DNA sequence required for efficient transcription termination in yeast. Cell 28, 563-573.

Zuker, M. (1989). On finding all suboptimal foldings of an RNA molecule. Science 244, 48-52.

Received 26 July 1996; revised 11 November 1996; accepted 2 December 1996. 